\title{
ORIGINAL ARTICLE Cellular and molecular basis for stress-induced depression
}

\author{
J-S Seo ${ }^{1}, \mathrm{~J} \mathrm{Wei}^{2}, \mathrm{~L} \mathrm{Qin}^{2}, \mathrm{Y} \mathrm{Kim}^{1}$, Z Yan ${ }^{2}$ and P Greengard ${ }^{1}$
}

Chronic stress has a crucial role in the development of psychiatric diseases, such as anxiety and depression. Dysfunction of the medial prefrontal cortex (mPFC) has been linked to the cognitive and emotional deficits induced by stress. However, little is known about the molecular and cellular determinants in MPFC for stress-associated mental disorders. Here we show that chronic restraint stress induces the selective loss of p11 (also known as annexin II light chain, S100A10), a multifunctional protein binding to 5-HT receptors, in layer II/III neurons of the prelimbic cortex (PrL), as well as depression-like behaviors, both of which are reversed by selective serotonin reuptake inhibitors (SSRIs) and the tricyclic class of antidepressant (TCA) agents. In layer II/III of the PrL, p11 is highly concentrated in dopamine D2 receptor-expressing $\left(D 2^{+}\right)$glutamatergic neurons. Viral expression of p11 in D2 ${ }^{+}$PrL neurons alleviates the depression-like behaviors exhibited by genetically manipulated mice with $\mathrm{D} 2^{+}$neuron-specific or global deletion of p11. In stressed animals, overexpression of p11 in D2 ${ }^{+}$PrL neurons rescues depression-like behaviors by restoring glutamatergic transmission. Our results have identified $\mathrm{p} 11$ as a key molecule in a specific cell type that regulates stress-induced depression, which provides a framework for the development of new strategies to treat stress-associated mental illnesses.

Molecular Psychiatry (2017) 22, 1440-1447; doi:10.1038/mp.2016.118; published online 26 July 2016

\section{INTRODUCTION}

Corticosteroid stress hormones serve as important regulators of cognitive and emotional processes by exerting complex effects in the central nervous system. ${ }^{1-6}$ Exposure to prolonged stress induces heightened vulnerability to anxiety, depression and other mood disorders. ${ }^{7-9}$

Among the multiple brain areas involved in cognition and emotion, the medial prefrontal cortex (mPFC), a region controlling higher-level 'executive' functions, is a primary target of stress hormones. ${ }^{5,10-12}$ Structural and functional changes induced by stress in the mPFC have been correlated with emotional disturbances in humans ${ }^{13-15}$ and rodents. ${ }^{3,11,12}$ Glutamate receptor-mediated synaptic transmission, which controls $\mathrm{mPFC}$ network activity, is crucial for mood and working memory. ${ }^{16,17}$ Repeated stress negatively influences mPFC-mediated cognitive processes by disturbing glutamatergic signaling in rats. ${ }^{18,19}$

A key question waiting to be answered is the molecular and cellular basis of stress-induced depression. In the brain, the multifunctional protein p11, which interacts with 5-HT receptors, ion channels, enzymes and chromatin-remodeling factors, has been found to be critically involved in depressionlike behaviors and/or antidepressant actions. ${ }^{20-23}$ p11 is enriched in distinct neuronal types, such as cholinergic neurons in nucleus accumbens, ${ }^{24}$ mossy cells and basket cells in dentate gyrus, ${ }^{20}$ and layer 5 corticostriatal projection neurons. ${ }^{25}$ However, it is largely unknown whether p11 in specific subtypes of neurons controls depressive phenotypes in response to chronic stress. In this study, we sought to investigate the anatomical distribution and identify the neuronal cell types of p11 in MPFC and to analyze its potential role in stress-induced depression. The synaptic mechanisms underlying the behavioral effects of p11 were also explored by examining glutamatergic signaling in mPFC of stressed animals.

\section{MATERIALS AND METHODS}

Animals

Eight transgenic mouse lines were generated and used for this study: p11-EGFPmice, ${ }^{21,22,26}$ D2-Cre mice, ${ }^{24,26}$ D2-tdT mice (D2-Cre line crossed with tdTomato line) ${ }_{1}^{20} \mathrm{p} 11 \mathrm{cKO}$ mice (D2-Cre line crossed with $\mathrm{p} 11^{\mathrm{f} / \mathrm{f}}$ line $^{27}$ ), p11 cKO-tdT mice (D2-Cre crossed with tdTomato line and p11 cKO line), p11 gKO mice (D2-Cre line crossed with p11 KO line ${ }^{21}$ ), p11 gKO-tdT mice (D2-Cre crossed with tdTomato line and p11 KO line) and D1-tdT-D2-eGFP mice (D1-Cre ${ }^{26}$ crossed with tdTomato line and D2-eGFP mice). The C57BL/6J mice and tdTomato reporter mice (Rosa26-CAG-tdTomatoloxp/+, 007908) were purchased from the Jackson Laboratory. We produced the progeny for each line by in vitro fertilization and embryo transfer techniques (Transgenic Facility, The Rockefeller University, New York, NY, USA).

All experiments were approved by The Rockefeller University Institutional Animal Care and Use Committee and were performed in accordance with the guidelines described in the National Institutes of Health Guide for the Care and Use of Laboratory Animals. Mice were housed in groups of up to five animals on a $12 \mathrm{~h}$ dark/light cycle at $22^{\circ} \mathrm{C}$ and maintained with rodent diet (Picolab, St. Louis, MO, USA) and water available ad libitum. Male mice were used for all experiments.

\section{Restraint stress and antidepressant treatments}

The restraint stress treatment was performed as previously described. ${ }^{20,28}$ Briefly, mice were housed two per cage and individually placed head-first into well-ventilated $50 \mathrm{ml}$ polypropylene conical tubes, which were then plugged with a $4.5-\mathrm{cm}$-long middle tube, and finally tied with a cap of the $50 \mathrm{ml}$ tube. After each session of restraint stress, the mice were returned to their home environment, in which they were housed in pairs in normal plastic cages with free access to food and water. From the next day after the last restraint session, imipramine, fluoxetine and escitalopram were administered by a daily I.P. injection $\left(20 \mathrm{mg} \mathrm{kg}^{-1}\right.$ day $\left.^{-1}\right)$ for up to 4 weeks. Imipramine, fluoxetine and escitalopram were purchased from SigmaAldrich (St. Louis, MO, USA). Fluoxetine and escitalopram were dissolved in dimethylsulfoxide and then diluted in saline. Imipramine was dissolved in saline. Each drug was finally diluted in $100 \mu \mathrm{l} \mathrm{of} 0.9 \%$ saline and

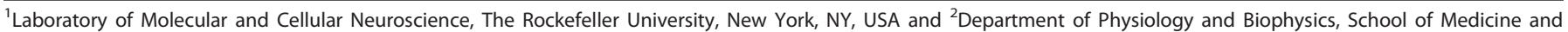

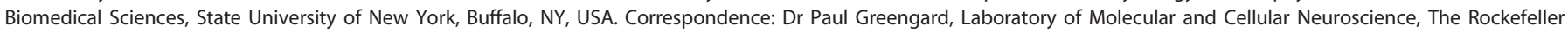
University, 1230 York Avenue, New York, NY 10065, USA.

E-mail: greengard@rockefeller.edu

Received 16 March 2016; revised 20 April 2016; accepted 27 April 2016; published online 26 July 2016 
administered at the dose indicated. Control groups were administered saline.

\section{Viruses}

For gene silencing, Lenti-GFP-shRNAmir (GFP-shRNA, RHS4348) and Lentip11-GFP-shRNAmir (p11-shRNA, VGM5524-99213741) viruses were purchased from Thermo Scientific (Waltham, MA, USA). For Cre-mediated recombination/inversion of the flanked p11 as double-floxed inverse (DIO; open reading frame) viruses, AAV vector production of the AAV2 serotype was performed by the University of Pennsylvania vector core. AAV2-EF1a-DIO-eYFP-WPRE-hGH was used as the control vector. AAV2EF1a-DIO-p11-WPRE-hGH vector construction for overexpression of p11 was made by Dr Jung-Hyuck Ahn (Ewha Womans University, Seoul, South Korea). Double-floxed AAV constructs were generated by insertion of the inverted p11 expression cassettes between double lox 2722 and lox P incompatible sites (DIO). In the absence of Cre expression, the p11 or eYFP were not produced. In the presence of Cre expression, the transgene will be FLEXed, leading to the expression of the p11 or eYFP. The titers (genome copies per milliliter) of the AAVs were as follows: 4.04e12 for AAV2-EF1a-DIO-eYFP-WPRE-hGH (AAV_eYFP) and 3.64e12 for AAV2-EF1aDIO-p11-WPRE-hGH (AAV_p11).

\section{Stereotaxic surgery}

All stereotaxic injections were carried out on an Angle Two stereotaxic frame for mouse with motorized nanoinjector (Leica, Buffalo Grove, IL, USA). Ten-week-old male mice were anesthetized with ketamine and xylazine and stereotaxically injected with Lenti-GFP-shRNA, Lenti-p11-GFPshRNA, AAV2-EF1a-DIO-eYFP-WPRE-hGH and AAV2-EF1a-DIO-p11-WPRE$\mathrm{hGH}$ into the layer II/II PrL (AP: $1.98 \mathrm{~mm}$; $\mathrm{ML}: \pm 0.12 \mathrm{~mm}$; and $\mathrm{DV}$ : $-2.21 \mathrm{~mm}$ from bregma). The total injection volume was $0.5 \mu \mathrm{l}$. All injections were performed at a rate of $0.15 \mu$ min $^{-1}$ using Hamilton syringes (33 gauge; Reno, NV, USA) and the needle was kept in place for an additional five minutes. After 14 days of injection, depression-like behavioral tests and electrophysiological recording were performed.

\section{Behavioral assessments}

As previously described, ${ }^{20,28}$ all behavioral tests were performed during the light cycle in a dedicated sound-proof behavioral facility by experimenters blind to treatment- and genotype information. Mice were brought to the testing room $30 \mathrm{~min}$ before the start of each behavioral test and remained in the same room through the test. At all times, sound was masked with 60-65 dB white noise.

Tail suspension test. Mice were suspended individually by their tails. The rod was fixed $50 \mathrm{~cm}$ above the surface of a table covered with a safety mat in a sound-isolated room. The tip of the tail was fixed using adhesive Scotch tape; the duration of the test was $5 \mathrm{~min}$. The test session was videotaped and immobility scored by using automated tail suspension test/forced swin test (TST/FST) analysis software from Clever Systems (Reston, VA, USA).

Forced swim test. In brief, mice were placed in a glass cylinder (height: $30 \mathrm{~cm}$, diameter: $16 \mathrm{~cm}$ ) containing water at $24{ }^{\circ} \mathrm{C}$ and a depth of $14 \mathrm{~cm}$, so that they could neither escape nor touch the bottom. Mice were forced to swim for $6 \mathrm{~min}$. The animals were habituated for the first $1 \mathrm{~min}$ and behavior was monitored over the next $5 \mathrm{~min}$. A 6 min test session was videotaped and immobility scored by using automated TST/FST analysis software from Clever Systems.

Sucrose preference test. Mice were presented with two water bottles. After habituation for 1 day, mice were given a free choice between two bottles, containing tap water or $2 \%$ sucrose solution. To prevent a possible effect of drinking behavior, the left/right location of the bottles was switched every day. The consumption of water and sucrose solution was measured daily for 3 days by weighing the bottles. The sucrose preference was calculated as the ratio of consumed sucrose solution to consumed water.

Novelty-suppressed feeding test. After 24- $\mathrm{h}$ food deprivation (water was provided ad libitum), mice were assayed by novelty-supressed feeding (NSF) test, At the end of this time, a single $2 \times 2 \mathrm{~cm}$ oval food pellet was placed on a circular piece of white filter paper $(150 \mathrm{~mm}$ diameter) positioned in the center of the open field $(40 \times 40 \times 40 \mathrm{~cm})$. Each mouse was placed in a corner of the open field. The latency to first bite the laboratory chow pellet and consumption over 15 min were recorded. Immediately after the mouse began to eat the chow, the tested animal was placed in its home cage alone with a weighed piece of chow for $30 \mathrm{~min}$. At the end of this period, the amount of food consumed was determined by weighing the piece of chow.

Locomotion test. Locomotor activity was measured in the open field of a Plexiglas chamber $(40 \times 40 \times 40 \mathrm{~cm})$. Each mouse was placed in the corner of the open field, and locomotion was recorded for the indicated period for $30 \mathrm{~min}$. An automated Superflex software (Accuscan Instruments, Columbus, OH, USA) was used to measure the total distance traveled across a session. The measures were automatized using two rows of infrared photocells placed 20 and $50 \mathrm{~mm}$ above the floor, spaced $31 \mathrm{~mm}$ apart. Photocell beam interruptions were recorded on a computer using the Superflex software (Accuscan Instruments).

\section{Immunohistochemistry}

Brains were perfused transcardially with cold phosphate-buffered saline (PBS), followed by $4 \%$ paraformaldehyde and postfixed in the same solution overnight at $4{ }^{\circ} \mathrm{C}$. The brains were coronally cut into $40-\mu \mathrm{m}$-thick sections with a vibratome (VT $1000 \mathrm{~S}$, Leica). Free-floating sections were washed three times with $0.1 \mathrm{M}$ PBS containing $0.1 \%$ Triton X-100 in phosphate buffered saline-Tween 20 (PBS-T), pH 7.4, for 15 min each time and permeabilized with PBST in $2 \%$ normal goat serum, $2 \%$ normal horse serum and $2 \%$ bovine serum albumin for $1 \mathrm{~h}$. After blocking, sections were incubated with the primary antibodies diluted in the blocking buffer. The immunohistochemistry was done using the following antibodies: anti-p11 (goat polyclonal, 1:200, R\&D Systems, Minneapolis, MN, USA), anti-eGFP (chicken polyclonal, 1:500, Abcam, Cambridge, MA, USA), anti-CaMKII (rabbit polyclonal, 1:500, Santa Cruz, Dallas, TX, USA) and anti-GAD67 (mouse monoclonal 1:1000, Millipore, Billerica, MA, USA). After $24 \mathrm{~h}$ incubation, sections were washed, and incubated with Alexa-fluorconjugated secondary antibodies (1:500, Invitrogen, Carlsbad, CA, USA). Slices were washed three more times in PBS-T for 15 min each and mounted with Vectashield mounting medium with DAPI (Vector Laboratories, Burlingame, CA, USA) onto microscope slides. All the sections were examined under a Zeiss LSM710 confocal microscope or wide-field fluorescence microscope (Zeiss, Jena, Germany). All histology findings were confirmed in at least five different animals.

\section{Cell counting}

The number of eGFP, tdTomato, p11, CaMKII or GAD67-immunolabeled neurons was quantified with ImageJ software (NIH). Three to five coronal sections per animal were quantified and averaged for each animal. Fluorescence images for obtaining layer II/III PrL were acquired using a Zeiss LSM710 confocal microscope with a $\times 40 / 0.50$ NA objective $\left(45176.65 \mathrm{um}^{2} ; 262144\right.$ pixels). Background autofluorescence was accounted for by applying an equal cutoff threshold to all images. All imaging and analyses were performed blind to the experimental conditions. A one-way analysis of variance followed by Newman-Keuls post hoc test multiple comparisons or one-sample $t$-tests were used to analyze data and later graphed using Microsoft Excel or Prism Software (GraphPad, La Jolla, CA, USA).

\section{Western blot analysis}

Tissue samples were obtained from the PrL of the mice. Tissue samples were homogenized in RIPA buffer (Sigma-Aldrich) supplemented with a protease inhibitor cocktail (Complete-EDTAfree; Roche) and a phosphatase inhibitor cocktail (PhosStop, Roche). Protein concentrations were determined using a BCA assay (Thermo Scientific). A total of $30 \mu \mathrm{g}$ of protein were denatured in Laemmli sample buffer at $95^{\circ} \mathrm{C}$ for $5 \mathrm{~min}$ and separated by SDS-polyacrylamide gel electrophoresis using 4-20\% tris-glycine gel (Life Technologies, Gaithersburg, MD, USA). After transfer of proteins to nitrocellulose membranes, blots were blocked in $5 \%$ non-fat milk for $1 \mathrm{~h}$ at room temperature and incubated with the respective primary antibody at $4{ }^{\circ} \mathrm{C}$ overnight. Primary antibodies were as follows: anti-p11 (goat polyclonal, 1:500, R\&D Systems); and anti- $\beta$ actin (mouse monoclonal, 1:5000, Abcam). Primary antibodies were detected using either HRP-linked donkey anti-goat lgG (1:2000, Santa Cruz) or HRP-linked sheep anti-mouse IgG (1:10000, GE Healthcare, Madison, WI, USA) together with Western Lightning Plus-ECL (Perkin Elmer, Norwalk, CT, USA). Signals were quantified with ImageJ software (NIH). 


\section{Quantitative RT-PCR}

Reverse transcription was performed with $1 \mu \mathrm{g}$ of total RNA using ImProm-II Reverse Transcription System (Promega, Madison, WI, USA) with Oligo dT primer according to the manufacturer's protocol. A total of $10 \mathrm{ng}$ of CDNA was used for each quantitative PCR reaction and all samples were run in triplicate. Quantitative PCR was carried out using an Applied Biosystems $7900 \mathrm{HT}$ system (Foster City, CA, USA). Taqman Universal PCR Master Mix (Life Technologies, Foster City, CA, USA) was used for all analyses. Taqman gene expression assays (FAM) from Life Technologies were as follows: 111 (Mm00501457_m1) and Gapdh (Mm99999915_g1). All data were normalized to TaqMan Rodent GAPDH Control, and relative expression levels between conditions were calculated by the comparative $C T(2-\Delta \Delta C T)$ method.

\section{Electrophysiological recordings}

As previously described, ${ }^{19,29}$ whole-cell voltage-clamp recording technique was used to measure synaptic currents in layer II/III PrL D2 ${ }^{+}$neurons. After chronic restraint stress treatment with or without viral (AAV2-EF1a-DIOeYFP-WPRE-hGH or AAV2-EF1a-DIO-p11-WPRE-hGH) injection into the layer II/III PrL, D2-tdT mice (D2-Cre line crossed with tdTomato line) or p11 cKO-tdT mice (D2-Cre crossed with tdTomato line and p11 cKO line) were used for electrophysiological experiments. Red fluorescent $\mathrm{D}^{+}$ neurons were selected for recordings. Mouse slices $(300 \mu \mathrm{m})$ were positioned in a perfusion chamber attached to the fixed stage of an upright microscope (Olympus, Tokyo, Japan) and submerged in continuously flowing oxygenated artificial cerebrospinal fluid (in mM: $130 \mathrm{NaCl}$, $26 \mathrm{NaHCO}_{3}, 1 \mathrm{CaCl}_{2}, 5 \mathrm{MgCl}_{2}, 3 \mathrm{KCl}, 1.25 \mathrm{NaH}_{2} \mathrm{PO}_{4}, 10$ glucose, $\mathrm{pH} 7.4$, $300 \mathrm{mOsm})$. Bicuculline $(10 \mu \mathrm{m})$ and $\mathrm{CNQX}(20 \mu \mathrm{m})$ were added for $\mathrm{N}$ methyl-D-aspartate receptor-mediated excitatory postsynaptic current (NMDAR-EPSC) recordings. Bicuculline and D-APV $(50 \mu \mathrm{m})$ were added in a-amino-3-hydroxy-5-methyl-4-isoxazolepropionic acid receptor-mediated excitatory postsynaptic current (AMPAR-EPSC) recordings. Patch electrodes contained an internal solution (in $\mathrm{mm}$ ): $130 \mathrm{Cs}$-methanesulfonate, $10 \mathrm{CsCl} 4$ $\mathrm{NaCl}, 10$ HEPES, $1 \mathrm{MgCl}_{2}$, 5 EGTA, 2 QX-314, 12 phosphocreatine, 5 MgATP, $0.2 \mathrm{Na}_{3}$ GTP, 0.1 leupeptin, pH 7.2-7.3, 265-270 mOsm. Layer II/III PrL D2 ${ }^{+}$ neurons were visualized with a $\times 40$ water-immersion lens and recorded with the Multiclamp 700A amplifier (Molecular Devices, Sunnyvale, CA, USA). Evoked EPSC were generated with a pulse from a stimulation isolation unit controlled by an S48 pulse generator (Grass Technologies, West Warwick, RI, USA). A bipolar stimulating electrode (FHC, Bowdoin, ME, USA) was placed $\sim 100 \mu \mathrm{m}$ from the neuron under recording. For NMDAREPSC, the cell (clamped at $-70 \mathrm{mV}$ ) was depolarized to $+40 \mathrm{mV}$ for $3 \mathrm{~s}$ a

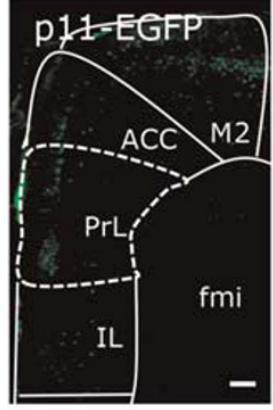

b
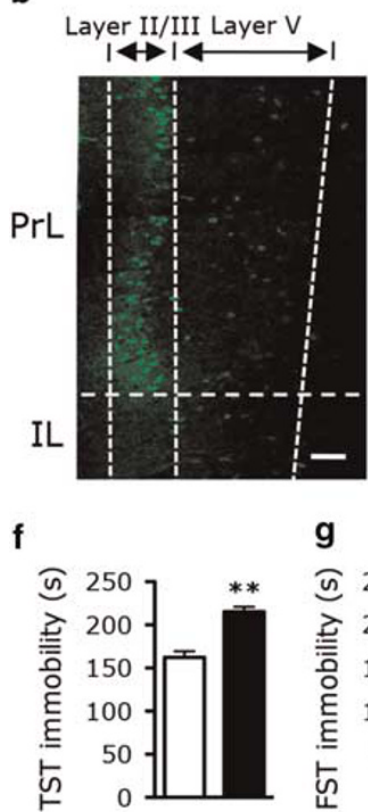
g
C

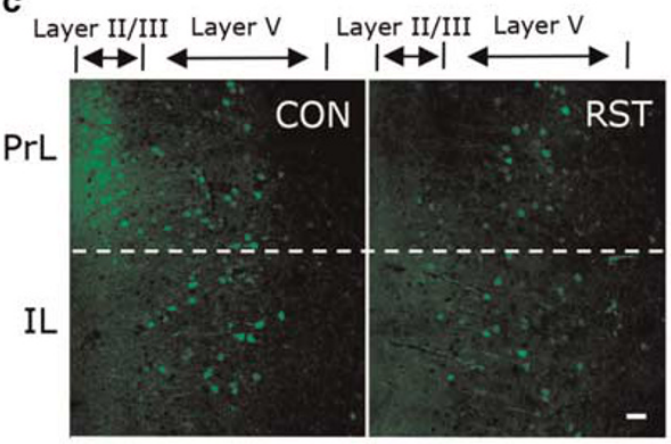

d

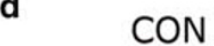

RST
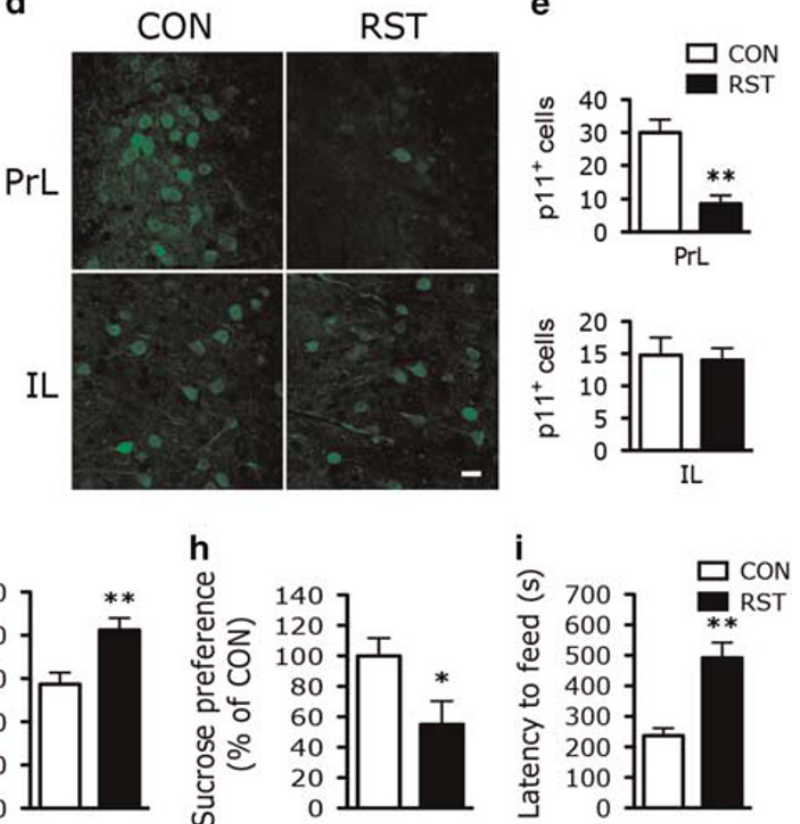

Figure 1. Chronic stress induces loss of p11 in PrL, as well as depression-like behavior. (a) Immunofluorescence image illustrating p11-positive cells $\left(E_{G F P}^{+}\right)$in mPFC. Scale bar, $500 \mu \mathrm{m}$. (b) High-magnification image of p11 expression in different layers of PrL and IL. Scale bar, $100 \mu \mathrm{m}$. (c) p11-expressing cells in the PrL and IL from control (CON) and chronic restraint stressed (RST) mice. Scale bar, $40 \mu \mathrm{m}$. (d) High-magnification images of p11 expression in PrL layer II/III and IL layer V from control and stressed mice. Scale bar, $25 \mu \mathrm{m}$. (e) Quantification of p11-expressing cells in PrL (layer II/III) and IL (layer V) from control and stressed mice ( $n=5$ per group). (f-i) Chronic stress-induced depression-like behaviors measured by immobility time in TST (f), FST (g), the ratio of sucrose to water consumption in (h) and the latency to feed in NSF test (i) ( $n=24$ per group). ${ }^{*} P<0.05,{ }^{*} P<0.01$, two-tailed $t$-test. ACC, anterior cingulate cortex; FST, forced swin test; fmi, forceps minor of the corpus callosum; IL, infralimbic cortex; M2, motor cortex 2; NSF, novelty-supressed feeding; PrL, prelimbic cortex; SPT, sucrose preference test. Data are means \pm s.e.m. 
before stimulation to fully relieve the voltage-dependent $\mathrm{Mg}^{2+}$ block. Membrane potential was maintained at $-70 \mathrm{mV}$ for AMPAR-EPSC recordings. For input-output responses, EPSC was elicited by a series of pulses with different stimulation intensities (50-90 $\mu \mathrm{A})$ delivered at $0.033 \mathrm{~Hz}$. For paired-pulse ratios, AMPAR-EPSC was evoked by double pulses with various intervals $(0.02-0.4 \mathrm{~s})$. Data analyses were performed with Clampfit (Axon instruments, Molecular Devices), Kaleidagraph software (Albeck, Synergy Software, Reading, PA, USA) and Prism software (GraphPad).

\section{Statistics}

Two-sample comparisons were performed using the Student's $t$-test, while multiple comparisons were made using one-way analysis of variance followed by a Newman-Keuls post hoc test and two-way analysis of variance by a Bonferroni post hoc test to compare selected pairs of data. PRISM software (GraphPad Software) was used to perform statistical analyses. All data are presented as the mean \pm s.e.m.

\section{RESULTS}

Chronic stress induces the loss of p11 expression in PrL and depression-like behaviors

To identify the neuronal types of p11 in mPFC that may be involved in stress-induced depression, we first examined the anatomical and cellular distribution of p11 using p11 promoterdriven EGFP ( $p 11-E G F P)$ mice. GFP immunofluorescence showed that $\mathrm{p} 11$ was present in all three subregions of $\mathrm{MPFC}$, anterior cingulate cortex, prelimbic cortex (PrL) and infralimbic cortex (IL), but that it was most abundant in layer II/III pyramidal neurons of PrL (Figures 1a and b).

Next, we investigated whether p11 expression in mPFC, especially PrL and IL (rodent homolog of human orbitofrontal corte $\left.{ }^{30}\right)$, was altered by stress. Mice exposed to chronic restraint stress (RST, $2 \mathrm{~h} /$ day, 14 days) ${ }^{20,28}$ exhibited significantly reduced p11 expression in layer II/III PrL neurons, but not in IL neurons, compared with the control group (Figures 1c-e).

In parallel, we also examined behavioral outcomes of chronic stress. Compared with the control group, mice exposed to RST exhibited significantly increased immobility in TST (Figure 1f) and FST (Figure 1g), two measurements of helplessness and hopelessness. Stressed mice also showed anhedonia in sucrose preference test (SPT) (Figure 1h) and anxiety in NSF (Figure 1i), while food consumption and locomotor activity were not altered (data not shown). These data indicate that the chronic stress paradigm induces depression-like behaviors.
To determine whether the stress-induced loss of PrL p11 is responsible for depression-like behaviors, we knocked down p11 expression by injecting p11 shRNA lentivirus into PrL (Supplementary Figure S1a). The p11 shRNA induced a potent suppression of p11 expression in PrL (Supplementary Figure S1b). The p11 shRNA-injected mice exhibited significantly increased immobility in both TST and FST (Supplementary Figures S1c and d), indicating that the loss of p11 from PrL contributes to the manifestation of depression-like behaviors.

Antidepressants restore p11 levels in PrL and alleviate stressinduced depression

To further determine the role of $\operatorname{PrL} \mathrm{p} 11$, we investigated the impact of antidepressant treatment on stress-induced changes in p11 expression and depression-like behaviors. Western blotting and quantitative $P C R$ results revealed that the levels of $\mathrm{p} 11$ protein and mRNA in PrL were reduced in chronically stressed mice, which were reversed by 2-week treatment with three distinct antidepressants, imipramine (TCA), fluoxetine (selective serotonin reuptake inhibitors (SSRIs)) and escitalopram (SSRI) (Figures $2 \mathrm{a}$ and b). These antidepressants also rescued the depression-like behaviors in TST, FST, SPT and NSF (Figures 2c-f).

Chronic stress-induced depression-like behaviors are long lasting, but can be reversed over time. ${ }^{28,31}$ Therefore, we also examined the natural recovery of stress-induced changes in $\mathrm{p} 11$ expression and behavior. At 14 days post stress, the level of $p 11$ in PrL was significantly lower than control animals, but at 90 days post stress, the level of $\mathrm{p} 11$ in PrL was restored to the control level (Supplementary Figures S2a and b). In parallel, the depression-like behaviors measured by TST and FST disappeared 90 days, but not 14 days, post stress (Supplementary Figures S2c and d). More detailed time courses showed that the recovery of $\mathrm{p} 11$ expression and behaviors were well correlated (Supplementary Figure S2).

The expression of p11 in D2R-containing glutamatergic PrL neurons determines depression-like behavior

Given the heterogeneity of PrL neurons, we sought to determine which neuronal subtypes were involved in stress-induced depression. Immunofluorescence staining revealed that, in layer II/III PrL, p11 was present in most ( 88\%) D2 dopamine receptorexpressing $\left(\mathrm{D}^{+}\right)$neurons (Figure 3a; Supplementary Figures S3a-C; and Supplementary Table S1-3), but only in a few ( 8\%) D1 dopamine receptor-expressing $\left(\mathrm{D} 1^{+}\right)$neurons (Supplementary a
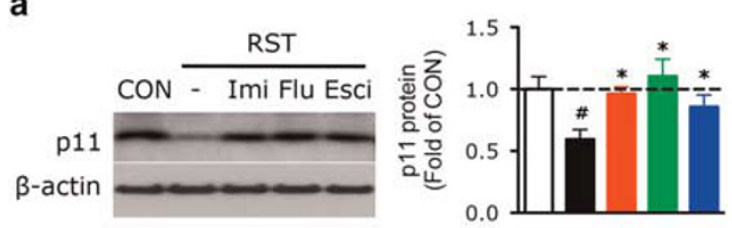

b
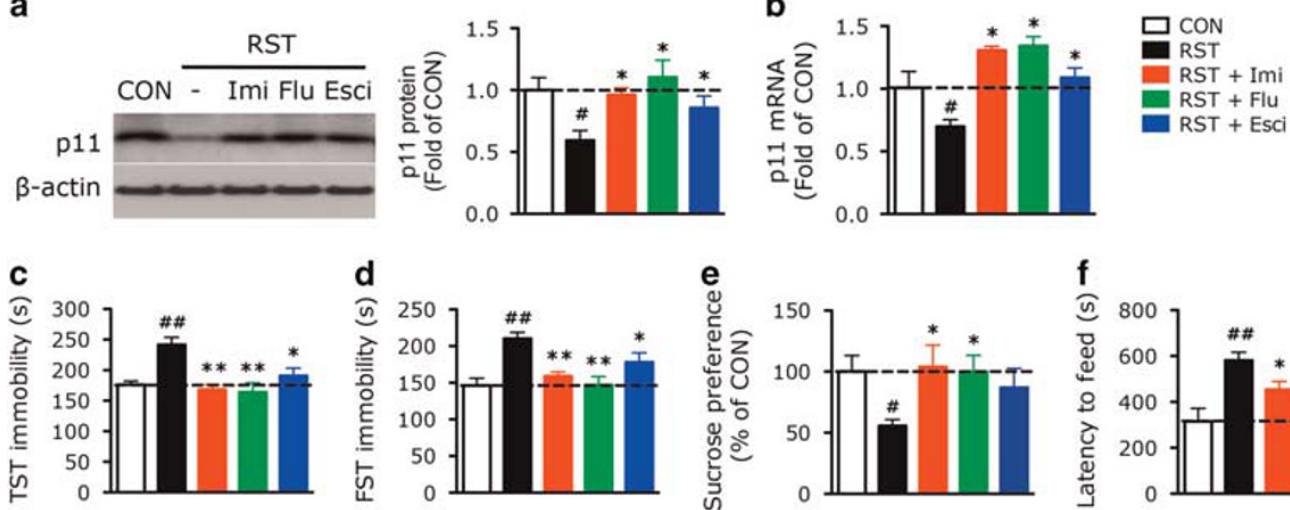
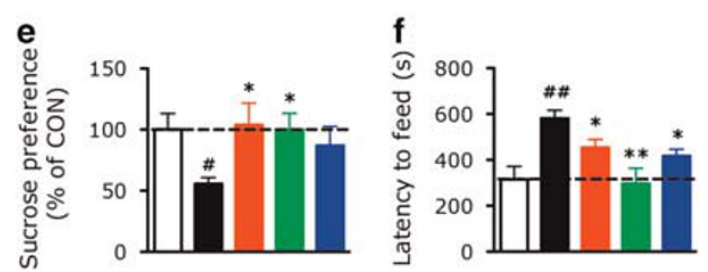

Figure 2. Antidepressants restore p11 levels in PrL and alleviate stress-induced depression. (a,b) Western blot measurement of p11 protein (a) and qPCR measurement of p11 mRNA (b) in PrL from control and stressed mice with or without antidepressant treatments $(\mathbf{a}, n=10$ CON, $n=12$ for each group of RST, RST+Imi, RST+Flu, RST+Esci; $\mathbf{b}, n=6$ per group). (c-f) Depression-like behaviors in control and stressed mice with or without antidepressant treatments, as measured by TST (c), FST (d), SPT (e) and NSF (f) $(n=10$ CON, $n=12$ for each group of RST, RST+Imi, RST+Flu, RST+Esci). ${ }^{\#} P<0.05$, \#\# $P<0.01$, compared with CON; ${ }^{*} P<0.05$ and ${ }^{* *} P<0.01$, compared with RST, one-way analysis of variance. Imi, imipramine; Flu, fluoxetine; FST, forced swin test; Esci, escitalopram; NSF, novelty-supressed feeding; PrL, prelimbic cortex; qPCR, quantitative PCR; SPT, sucrose preference test; TST, tail suspension test. Data are means \pm s.e.m. 
Figure S3a; Supplementary Table S1). Most ( 88\%) of the $\mathrm{p} 11^{+}$/ $\mathrm{D}^{+}$neurons in layer II/III PrL also expressed $\mathrm{Ca}^{2+} /$ calmodulindependent kinase II (CaMKII, Supplementary Figure S3b; and Supplementary Table S2), but not glutamic acid decarboxylase 67 (GAD67, Supplementary Figure S3c; and Supplementary Table S3), indicating that they are glutamatergic neurons.

To determine the role of p11 in layer II/III PrL D2 ${ }^{+}\left(\mathrm{D} 2^{+} \mathrm{PrL}\right)$ neurons, we generated conditional knockout mice with p11 deletion selectively in $\mathrm{D} 2^{+}$neurons $\left(\mathrm{p} 11 \mathrm{cKO}\right.$; D2-Cre $x \mathrm{p} 11^{\mathrm{f} / \mathrm{f}}$ ). The p11 cKO mice displayed depression-like behaviors as demonstrated by increased immobility in TST and FST (Figures $3 b$ and c), and anxiety-like behavior as indicated by increased latency to feed in NSF (Figure 3e). To specifically manipulate p11 expression in $\mathrm{D}^{+}$PrL neurons, we injected the Cre-dependent p11 overexpression virus (AAV_p11; AAV2-EF1a-DIO-p11-WPRE-hGH) to layer II/III PrL of p11 cKO mice, followed by behavioral measurements. As shown in Figures 3b-e, the depressive phenotypes of p11 cKO mice in TST, FST, SPT and NSF were all rescued by viral expression of $\mathrm{p} 11$ in $\mathrm{D}^{+}$PrL neurons.

Global p11 knockout (p11 gKO; D2-Cre x p11 KO) mice also manifested depressive behaviors in TST, FST, SPT and NSF (Figures $3 \mathrm{~g}-\mathrm{j}$ ), consistent with previous reports on p11 KO mice. ${ }^{21,24}$ Viral expression of p11 (AAV_p11) in D2 ${ }^{+}$PrL neurons of p11 gKO mice (Figure $3 \mathrm{f}$ ) rescued all the depression-like phenotypes (Figures $3 \mathrm{~g}-\mathrm{j}$ ). Collectively, these data indicate that restoration of $\mathrm{p} 11$ expression in $\mathrm{D} 2^{+} \operatorname{PrL}$ neurons is sufficient for the reversal of depressive behaviors induced by global deletion of $\mathrm{p} 11$.

p11 overexpression in D2R-containing PrL neurons ameliorates stress-induced behavioral and synaptic deficits

Given the loss of PrL p11 in stressed animals (Figures 1 and 2), we examined whether restoring $\mathrm{p} 11$ in $\mathrm{D} 2^{+} \mathrm{PrL}$ neurons could reverse stress-induced depression. TST, FST, SPT and NSF indicated that, compared with control D2-Cre mice [CON(D2)], chronically stressed D2-Cre mice [RST(D2)] exhibited depression-like behaviors, which were reversed by injecting AAV_p11, but not AAV_eYFP, to layer II/III PrL after stress (Figures 4a-d). Interestingly, overexpressing AAV p11 in layer II/III PrL of control mice [CON (D2)] induced anti-depression-like behaviors (Figures $4 a$ and b). These data further confirm that $\mathrm{p} 11$ in $\mathrm{D} 2^{+}$PrL neurons contributes to stress-induced depression.

To address the potential mechanisms underlying the role of $\mathrm{p} 11$ in stress-induced depression, we investigated the impact of p11 on PrL glutamatergic transmission, which plays a key role in regulating stress responses and emotional processes. ${ }^{5,6,12}$ We first

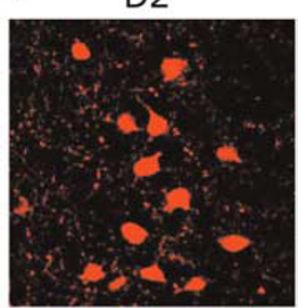

p11

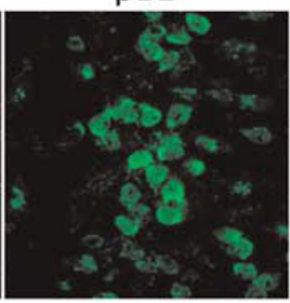

Merge

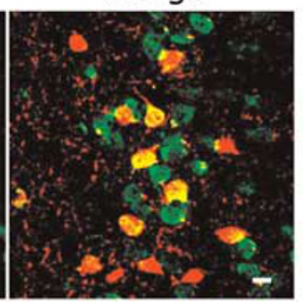

f

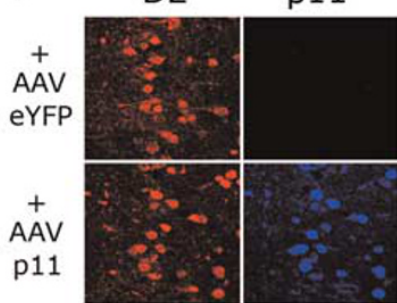

eYFP

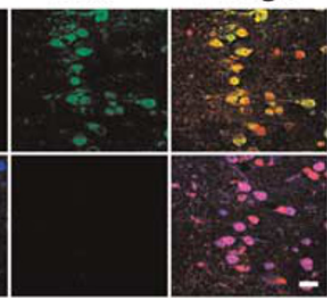

WT+AAV_eYFP

WT+AAV_p11

b
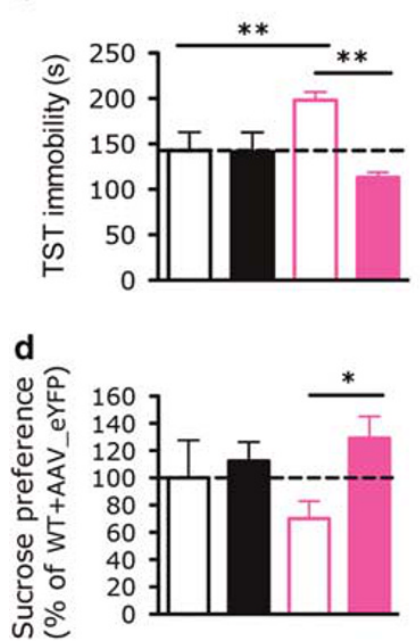

p11 cKO+AAV_eYFP p11 cKO+AAV_p11

C

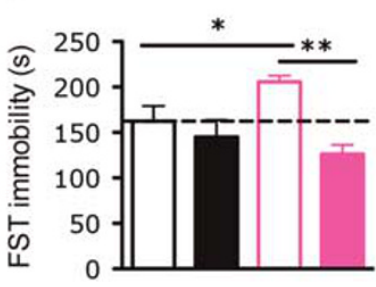

e

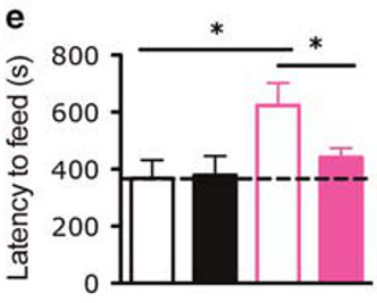

g

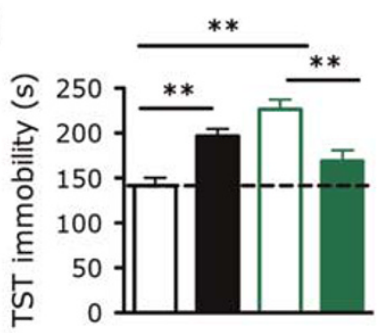

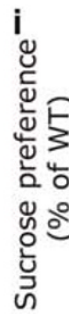

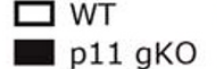

p11 gKO+AAV_eYFP p11 gKO+AAV_p11

h
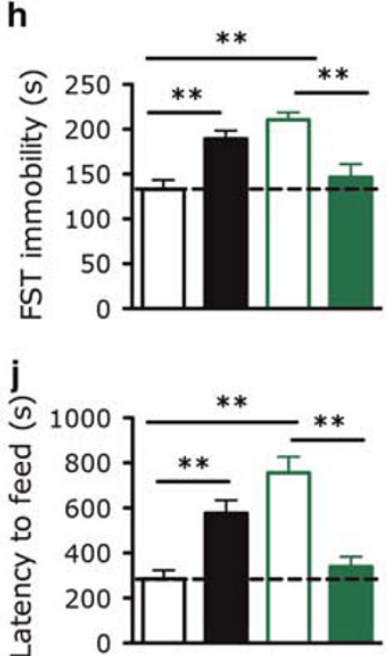

Figure 3. $\mathrm{p} 11$ in D2R-containing PrL neurons determines depression-like behavior. (a) Co-localization of dopamine D2 receptor (D2, red) and p11 (green) in layer II/III PrL neurons from D2-tdT (D2-Cre x tdTomato) mice. Scale bar, $10 \mu \mathrm{m}$. (b-e) Depression-like behaviors in wild-type (WT, $\mathrm{p} 11^{\mathrm{f} / \mathrm{f}}$ ) and D2 $2^{+}$neuron-specific conditional p11 knockout (p11 cKO, D2-Cre x p11 f/f) mice with the expression of AAV-DIO-p11 (AAV_p11) or AAV-DIO-eYFP (AAV_eYFP) in D2R-containing PrL neurons, as measured by TST (b), FST (c), SPT (d) and NSF (e) ( $n=6$, WT+AAV_eYFP; $n=6$, WT + AAV_p11; $n=12$, p11 cKO+AAV_eYFP; $n=12$, p11 cKO+AAV_p11). (f) Immunofluorescence images of p11 (blue) and eYFP (green) in global p11 knockout (p11 gKO-tdT, D2-Cre $x$ tdTomato $x$ p11 KO) mice with the injection of AAV_p11 or AAV_eYFP into layer II/III PrL. Scale bar, $20 \mu \mathrm{m}$. (g-j) Depression-like behaviors in WT and p11 gKO (D2-Cre x p11 KO) mice with the injection of AAV_p11 or AAV_eYFP into layer II/III PrL, as measured by TST (g), FST (h), SPT (i) and NSF (j) $\left(n=15, \mathrm{WT} ; n=16, \mathrm{p} 11 \mathrm{gKO} ; n=7, \mathrm{p} 11 \mathrm{gKO}+\mathrm{AAV} \_\right.$eYFP; $\left.n=6, \mathrm{p} 11 \mathrm{gKO}+\mathrm{AAV} \_\mathrm{p} 11\right)$. ${ }^{*} P<0.05$, ${ }^{*} P<0.01$, one-way analysis of variance. Data are means \pm s.e.m. FST, forced swin test; NSF, novelty-supressed feeding; PrL, prelimbic cortex; SPT, sucrose preference test; TST, tail suspension test. 


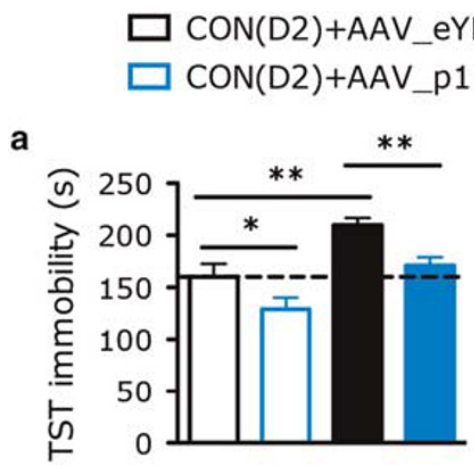

RST(D2)+AAV_eYFP

RST(D2)+AAV_p11

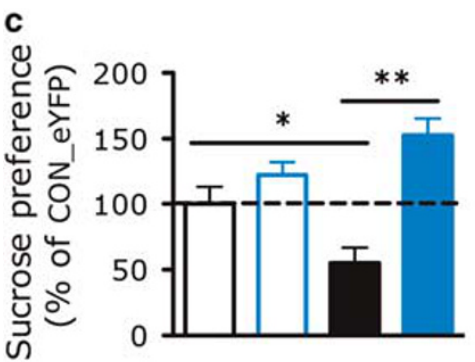

e

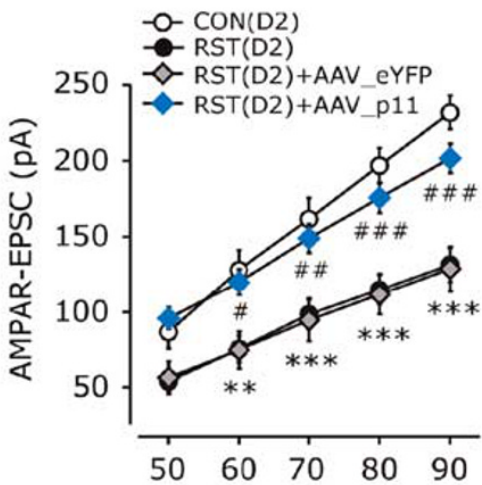

b

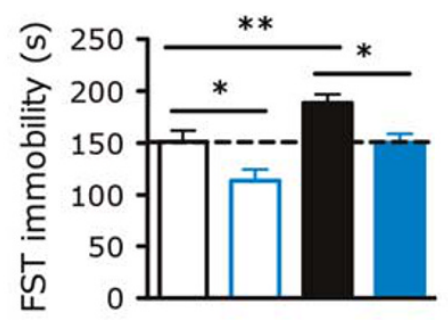

d

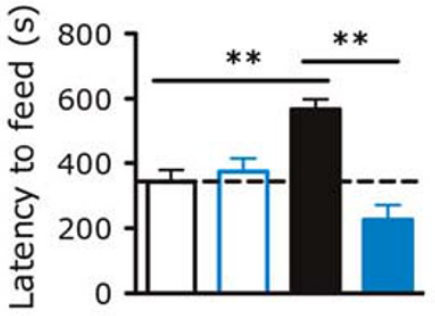

f

CON RST RST(D2) RST(D2)

(D2) (D2) + AAV_eYFP + AAV_p11

$50 \mu \mathrm{A} \sqrt{ } \mathrm{V}$

$\left.{ }^{70 \mu \mathrm{A}} \sqrt{ }\right]$

$90 \mu \mathrm{A}] / \sqrt{50 \mathrm{pAL}}]$

$20 \mathrm{~ms}$

Stimulation Intensity $(\mu \mathrm{A})$

g

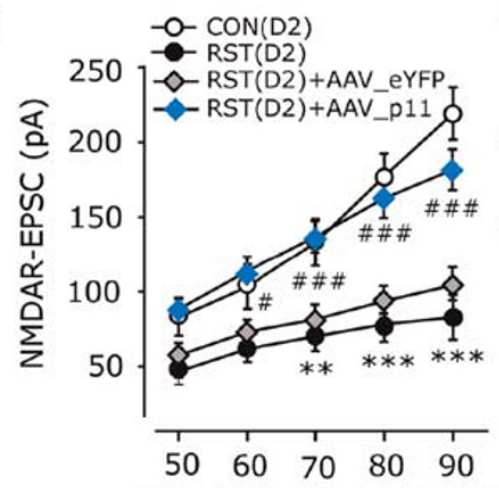

h
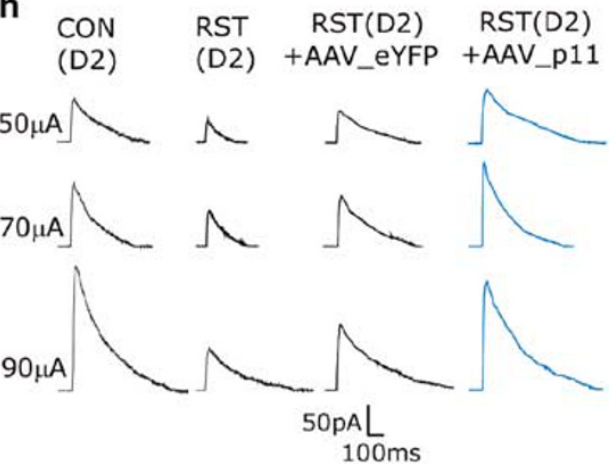

Stimulation Intensity $(\mu \mathrm{A})$

Figure 4. p11 overexpression in D2R-containing PrL neurons ameliorates stress-induced behavioral and glutamatergic synaptic deficits. (a-d) Depression-like behaviors in control and stressed D2-Cre mice with the expression of AAV_p11 or AAV_eYFP in D2R-containing PrL neurons, as measured by TST (a), FST (b), SPT (c) and NSF (d) $(n=10$ CON(D2)+AAV_eYFP, CON(D2)+AAV_p11; $n=12$ RST(D2)+AAV_eYFP; $n=14$ RST(D2) + AAV_p11). ${ }^{*} P<0.05,{ }^{*} P<0.01$, one-way analysis of variance. (e and $\left.\mathbf{g}\right)$ Summarized input-output curves of AMPAR-EPSC (e) and NMDA$\operatorname{EPSC}(\mathbf{g})$ in D2 ${ }^{+}$layer II/III PrL neurons from control mice (D2-tdT) and RST mice with PrL injection of AAV_eYFP or AAV_p11 (e, $n=21$ CON(D2), $n=26 \mathrm{RST}(\mathrm{D} 2), n=14 \mathrm{RST}(\mathrm{D} 2)+\mathrm{AAV}$ eYFP, $n=22 \mathrm{RST}(\mathrm{D} 2)+\mathrm{AAV} \_\mathrm{p} 11 ; \mathbf{g}, n=13 \mathrm{CON}(\mathrm{D} 2), n=13 \mathrm{RST}(\mathrm{D} 2), n=15 \mathrm{RST}(\mathrm{D} 2)+\mathrm{AAV}$ eYFP, $n=12 \mathrm{RST}$ (D2)+AAV_p11]. (f and h) Representative AMPAR-EPSC (f) and NMDAR-EPSC (h) traces in different groups. ${ }^{* *} P<0.01,{ }^{* * *} P<0.001, \mathrm{RST}(\mathrm{D} 2)$ vs CON(D2); ${ }^{\#} P<0.05,{ }^{\# \#} P<0.01,{ }^{\# \#} P<0.001, R S T(D 2)+A A V$ p1 1 vs RST(D2)+AAV eYFP, two-way analysis of variance (e and g). CON(D2), control D2-Cre mice; RST(D2), D2-Cre mice exposed to chronic restraint stress. Data are means \pm s.e.m. FST, forced swin test; NSF, novelty-supressed feeding; PrL, prelimbic cortex; SPT, sucrose preference test; TST, tail suspension test. 
examined the alteration of synaptic strength mediated by AMPARs and NMDARs in PrL neurons from stressed animals. The input-output curves of AMPAR-EPSC and NMDAR-EPSC were significantly decreased in layer II/III PrL D2 ${ }^{+}$neurons of stressed mice [RST(D2), Figures 4e-h], in parallel with the loss of p11 expression in these cells (Figures $1 \mathrm{c}$ and $\mathrm{d}$ and $2 \mathrm{a}$ and b). More importantly, overexpression of p11 (AAV_p11) in D2 ${ }^{+}$PrL neurons of stressed mice restored both AMPAR-EPSC and NMDAR-EPSC (Figures 4e-h). Manipulation of $\mathrm{p} 11$ in $\mathrm{D}^{+}$PrL neurons did not significantly alter the coefficient of variation of AMPAR-EPSC and NMDAR-EPSC, or paired-pulse ratio of AMPAR-EPSC (Supplementary Figures S4a-c), pointing to a postsynaptic locus for p11 effects on glutamatergic signaling. Collectively, these results show that p11 in D2 ${ }^{+}$PrL neurons may control stress-induced depression by regulating glutamatergic synaptic transmission.

\section{DISCUSSION}

Chronic stress causes the development of mood disorders including anxiety and depression. ${ }^{1-3,5,6}$ Our previous studies have implicated p11 as an important molecule involved in the etiology of depression and the mechanism of action of antidepressants. ${ }^{22}$ p11 was initially identified as a binding protein for serotonin receptors using a yeast two-hybrid screen. ${ }^{21,23}$ The level of $\mathrm{p} 11$ mRNA and protein is downregulated in the brain of depressed humans, suicide subjects and a mouse model of depression. ${ }^{21,32}$ In contrast, the level of p11 is increased by electro-convulsive therapy or chronic administration of monoaminergic antidepressants including SSRIs. ${ }^{20-22}$ p11 knockout mice exhibit depressionlike behaviors and reduced responses to SSRIs. ${ }^{21,22,25}$ Conversely, mice overexpressing p11 show antidepressant-like behaviors. ${ }^{21,24}$ SMARCA3, a chromatin-remodeling factor, is a binding partner of p11 and plays a central role in p11-dependent neurogenic and behavioral responses to SSRIs. Moreover, we have found that p11 binds to mGluR5 and increases the surface availability of the receptor, which provides a molecular mechanism underlying the antidepressant-like activity of mGluR5 antagonism. ${ }^{33}$ Two classes of antidepressant agents, including SSRIs, TCA as well as electroconvulsive therapy have each been shown to require p11 to achieve their therapeutic effects. ${ }^{21,22}$ It would be interesting to determine whether other classes of antidepressants, such as monoamine oxidase inhibitors also require p11 to achieve their therapeutic effects.

In the present study, by using a combination of genetic, molecular, cellular, electrophysiological and behavioral approaches, we have demonstrated that the depressive symptoms in stressed animals are closely associated with the reduction of p11 in layer II/III PrL. Antidepressants including SSRIs and TCA restore the expression of $\mathrm{p} 11$ in PrL, leading to the alleviation of stressinduced depression. Overexpressing $\mathrm{p} 11$ in layer II/III PrL D2 ${ }^{+}$ neurons $\left(\mathrm{D} 2^{+} \mathrm{PrL}\right)$ rescued depression-like behaviors in mice using two genetic models of $\mathrm{p} 11$ deletion ( $\mathrm{D} 2^{+}$neuron-specific or global), as well as chronic stress exposure. Thus, our results have identified $\mathrm{p} 11$ in $\mathrm{D}^{+}$PrL as a key molecular and cellular determinant in chronic stress-induced depression.

What are the potential mechanisms underlying the regulation of stress-induced depression by $\mathrm{p} 11$ in $\mathrm{D}^{+}$PrL? Our data demonstrate that AMPAR- and NMDAR-mediated glutamatergic transmission in $\mathrm{D} 2^{+} \operatorname{PrL}$ neurons is attenuated by chronic stress exposure, which is restored by $\mathrm{p} 11$ expression. Since the diminished glutamatergic signaling in PrL mediates cognitive and emotional disturbances in response to stress, ${ }^{6,12,17,19}$ the antidepression effects of p11 in stressed animals may be through the potentiation of glutamate receptor surface expression and synaptic function.

Medial prefrontal cortical pyramidal neurons project to several brain regions implicated in the pathophysiology of stress-induced psychiatric symptoms, ${ }^{2,3,5,12,34}$ such as nucleus accumbens, ${ }^{35-37}$ hippocampus, $^{38,39}$ lateral habenula, ${ }^{38,40,41}$ paraventricular nucleus ${ }^{42}$ of hypothalamus and basal lateral amygdala. ${ }^{38,43,44}$ The p11 regulation of glutamatergic transmission in layer II/III PrL D2 ${ }^{+}$ neurons may impact the synaptic drive to these subcortical regions, which mediates the depression-like behaviors through the integrated action of the emotional circuits.

Identification of key molecules in specific neuronal types that mediate the development of psychiatric disorders should enable the discovery of novel treatments. Our finding of a role for p11 in $\mathrm{D} 2^{+}$PrL neurons in the control of stress-induced depression provides a framework for the development of new strategies to treat stress-associated mental illnesses.

\section{CONFLICT OF INTEREST}

The authors declare no conflict of interest.

\section{ACKNOWLEDGMENTS}

We are grateful to Angus C Nairn, Marc Flajolet, Yotam Sagi and Lucian Medrihan for their helpful advice and discussion, and to Jung-Hyuck An for generating the AAV construct. We thank Xiaozhu Zhang for assistance with genotyping. We also thank the Rockefeller University Comparative Bioscience Center, Transgenics Services Laboratory and Genomics Resource Center. This work was supported by NIDA grant P01DA010044 (PG), NIMH grant P50MH090963 (PG), DOD/USAMRAA grant W81XWH-09-1-0402 (PG), the JPB Foundation (PG), the Fisher Center Foundation $(\mathrm{PG})$, the Leon Black Family Foundation (PG), NIH grants R01 MH108842 and R01 DA037618 (ZY).

\section{REFERENCES}

1 Caspi A, Sugden K, Moffitt TE, Taylor A, Craig IW, Harrington H et al. Influence of life stress on depression: moderation by a polymorphism in the $5-\mathrm{HTT}$ gene. Science 2003; 301: 386-389.

2 de Kloet ER, Joels M, Holsboer F. Stress and the brain: from adaptation to disease. Nat Rev Neurosci 2005; 6: 463-475.

3 Joels M, Karst H, Krugers HJ, Lucassen PJ. Chronic stress: implications for neuronal morphology, function and neurogenesis. Front Neuroendocrinol 2007; 28: 72-96.

4 Krishnan V, Nestler EJ. The molecular neurobiology of depression. Nature 2008; 455: 894-902.

5 McEwen BS. Physiology and neurobiology of stress and adaptation: central role of the brain. Physiol Rev 2007; 87: 873-904.

6 Pittenger C, Duman RS. Stress, depression, and neuroplasticity: a convergence of mechanisms. Neuropsychopharmacology 2008; 33: 88-109.

7 Cohen H, Kaplan Z, Matar MA, Loewenthal U, Zohar J, Richter-Levin G. Long-lasting behavioral effects of juvenile trauma in an animal model of PTSD associated with a failure of the autonomic nervous system to recover. Eur Neuropsychopharmacol 2006; 17: 464-477.

8 McEwen BS, Morrison JH. The brain on stress: vulnerability and plasticity of the prefrontal cortex over the life course. Neuron 2013; 79: 16-29.

9 Nemeroff CC. Early-life adversity, CRF dysregulation, and vulnerability to mood and anxiety disorders. Psychopharmacol Bull 2004; 38: 14-20.

10 Cerqueira JJ, Mailliet F, Almeida OF, Jay TM, Sousa N. The prefrontal cortex as a key target of the maladaptive response to stress. J Neurosci 2007; 27: 2781-2787.

11 Liston C, Miller MM, Goldwater DS, Radley JJ, Rocher AB, Hof PR et al. Stressinduced alterations in prefrontal cortical dendritic morphology predict selective impairments in perceptual attentional set-shifting. J Neurosci 2006; 26: 7870-7874.

12 Popoli M, Yan Z, McEwen BS, Sanacora G. The stressed synapse: the impact of stress and glucocorticoids on glutamate transmission. Nat Rev Neurosci 2012; 13: 22-37.

13 Drevets WC. Orbitofrontal cortex function and structure in depression. Ann NY Acad Sci 2007; 1121: 499-527.

14 Mayberg HS, Lozano AM, Voon V, McNeely HE, Seminowicz D, Hamani C et al. Deep brain stimulation for treatment-resistant depression. Neuron 2005; 45: 651-660.

15 Shin LM, Orr SP, Carson MA, Rauch SL, Macklin ML, Lasko NB et al. Regional cerebral blood flow in the amygdala and medial prefrontal cortex during traumatic imagery in male and female Vietnam veterans with PTSD. Arch Gen Psychiatry 2004; 61: 168-176.

16 Pessoa L. On the relationship between emotion and cognition. Nat Rev Neurosci 2008; 9: 148-158. 
17 Sanacora G, Treccani G, Popoli M. Towards a glutamate hypothesis of depression: an emerging frontier of neuropsychopharmacology for mood disorders. Neuropharmacology 2012; 62: 63-77.

18 Wei J, Xiong Z, Lee JB, Cheng J, Duffney LJ, Matas E et al. Histone modification of Nedd4 ubiquitin ligase controls the loss of AMPA receptors and cognitive impairment induced by repeated stress. J Neurosci 2016; 36: 2119-2130.

19 Yuen EY, Wei J, Liu W, Zhong P, Li X, Yan Z. Repeated stress causes cognitive impairment by suppressing glutamate receptor expression and function in prefrontal cortex. Neuron 2012; 73: 962-977.

20 Oh YS, Gao P, Lee KW, Ceglia I, Seo JS, Zhang X et al. SMARCA3, a chromatinremodeling factor, is required for p11-dependent antidepressant action. Cell 2013; 152: $831-843$

21 Svenningsson P, Chergui K, Rachleff I, Flajolet M, Zhang X, El Yacoubi M et al. Alterations in 5 -HT1B receptor function by $\mathrm{p} 11$ in depression-like states. Science 2006; 311: 77-80.

22 Svenningsson P, Kim Y, Warner-Schmidt J, Oh YS, Greengard P. p11 and its role in depression and therapeutic responses to antidepressants. Nat Rev Neurosci 2013; 14: 673-680.

23 Warner-Schmidt JL, Flajolet M, Maller A, Chen EY, Qi H, Svenningsson P et al. Role of $\mathrm{p} 11$ in cellular and behavioral effects of 5-HT4 receptor stimulation. J Neurosci 2009; 29: 1937-1946.

24 Warner-Schmidt JL, Schmidt EF, Marshall JJ, Rubin AJ, Arango-Lievano M, Kaplitt MG et al. Cholinergic interneurons in the nucleus accumbens regulate depression-like behavior. Proc Natl Acad Sci USA 2010; 109: 11360-11365.

25 Schmidt EF, Warner-Schmidt JL, Otopalik BG, Pickett SB, Greengard P, Heintz N. Identification of the cortical neurons that mediate antidepressant responses. Cell 2012; 149: 1152-1163.

26 Gong S, Zheng C, Doughty ML, Losos K, Didkovsky N, Schambra UB et al. A gene expression atlas of the central nervous system based on bacterial artificial chromosomes. Nature 2003; 425: 917-925.

27 Warner-Schmidt JL, Vanover KE, Chen EY, Marshall JJ, Greengard P. Antidepressant effects of selective serotonin reuptake inhibitors (SSRIs) are attenuated by antiinflammatory drugs in mice and humans. Proc Natl Acad Sci USA 2011; 108: 9262-9267.

28 Seo JS, Park JY, Choi J, Kim TK, Shin JH, Lee JK et al. NADPH oxidase mediates depressive behavior induced by chronic stress in mice. J Neurosci 2012; 32: 9690-9699.

29 Duffney $\amalg$, Zhong P, Wei J, Matas E, Cheng J, Qin L et al. Autism-like deficits in Shank3-deficient mice are rescued by targeting actin regulators. Cell Rep 2015; 11 : 1400-1413.

30 Wallis JD. Cross-species studies of orbitofrontal cortex and value-based decision-making. Nat Neurosci 2012; 15: 13-19.

31 Gray JD, Rubin TG, Hunter RG, McEwen BS. Hippocampal gene expression changes underlying stress sensitization and recovery. Mol Psychiatry 2014; 19: 1171-1178.
32 Anisman H, Du L, Palkovits M, Faludi G, Kovacs GG, Szontagh-Kishazi P et al. Serotonin receptor subtype and p11 mRNA expression in stress-relevant brain regions of suicide and control subjects. J Psychiatry Neurosci 2008; 33: 131-141.

33 Lee KW, Westin L, Kim J, Chang JC, Oh YS, Amreen B et al. Alteration by p11 of mGluR5 localization regulates depression-like behaviors. Mol Psychiatry 2015; 20: 1546-1556.

34 Arnsten AF. Stress signalling pathways that impair prefrontal cortex structure and function. Nat Rev Neurosci 2009; 10: 410-422.

35 Dias C, Feng J, Sun H, Shao NY, Mazei-Robison MS, Damez-Werno D et al. Beta-catenin mediates stress resilience through Dicer1/microRNA regulation. Nature 2014; 516: 51-55.

36 Lim BK, Huang KW, Grueter BA, Rothwell PE, Malenka RC. Anhedonia requires MC4Rmediated synaptic adaptations in nucleus accumbens. Nature 2012; 487: 183-189.

37 Ma YY, Lee BR, Wang X, Guo C, Liu L, Cui R et al. Bidirectional modulation of incubation of cocaine craving by silent synapse-based remodeling of prefrontal cortex to accumbens projections. Neuron 2014; 83: 1453-1467.

38 Hoover WB, Vertes RP. Anatomical analysis of afferent projections to the medial prefrontal cortex in the rat. Brain Struct Funct 2007; 212: 149-179.

39 Ramirez S, Liu X, MacDonald CJ, Moffa A, Zhou J, Redondo RL et al. Activating positive memory engrams suppresses depression-like behaviour. Nature 2015; 522: 335-339.

40 Li B, Piriz J, Mirrione M, Chung C, Proulx CD, Schulz D et al. Synaptic potentiation onto habenula neurons in the learned helplessness model of depression. Nature 2011; 470: 535-539.

41 Li K, Zhou T, Liao L, Yang Z, Wong C, Henn F et al. betaCaMKII in lateral habenula mediates core symptoms of depression. Science 2013; 341: 1016-1020.

42 Radley JJ, Arias CM, Sawchenko PE. Regional differentiation of the medial prefrontal cortex in regulating adaptive responses to acute emotional stress. J Neurosci 2006; 26: 12967-12976.

43 Adhikari A, Lerner TN, Finkelstein J, Pak S, Jennings JH, Davidson TJ et al. Basomedial amygdala mediates top-down control of anxiety and fear. Nature 2015; 527: 179-185.

44 Russo SJ, Nestler EJ. The brain reward circuitry in mood disorders. Nat Rev Neurosci 2013; 14: 609-625.

(i) This work is licensed under a Creative Commons AttributionBY NC ND NonCommercial-NoDerivs 4.0 International License. The images or other third party material in this article are included in the article's Creative Commons license, unless indicated otherwise in the credit line; if the material is not included under the Creative Commons license, users will need to obtain permission from the license holder to reproduce the material. To view a copy of this license, visit http:// creativecommons.org/licenses/by-nc-nd/4.0/

(c) The Author(s) 2017

Supplementary Information accompanies the paper on the Molecular Psychiatry website (http://www.nature.com/mp) 